\title{
La compasión como elemento clave de la práctica profesional en las instituciones de salud
}

\section{Autoras:}

María Blanco. Médico. Unidad de Investigación en Salud Reproductiva (UNISAR), Escuela de Enfermería Dra. Gladys Román de Cisneros, Valencia, Venezuela. ORCID: 0000-0002-4420-406X. Mail: delosangeles100190@gmail.com

Berta Guevara. Doctora en Enfermería. Unidad de Investigación en Gerontología (UNINGER) Escuela de Enfermería Dra. Gladys Román de Cisneros, Valencia, Venezuela. ORCID: 0000-0002-2115-5779. Mail: bertaguevara958@gmail.com

Marianela Mejías. Doctora en Enfermería. Unidad de Investigación en Enfermería (UNIVE), Escuela de Enfermería Dra. Gladys Román de Cisneros, Valencia, Venezuela. ORCID: 0000-0001-6315-1920. Mail: nelamejias@hotmail.com

No existen conflicto de interés

No se contó con ningún tipo de financiamiento

Fecha de Recepción: 13 de Abril de 2021

Fecha Aceptación: 07 de Junio 2021

DOI: http://doi.org/10.22370/bre.61.2021.3039

\section{> Resumen}

La práctica de los profesionales de la salud se sustenta en una relación interpersonal, requiere de sensibilidad y empatía, no es una simple actividad mecánica o un actuar sobre el paciente o familia, es una actividad donde se involucran las partes en la búsqueda de alivio del sufrimiento y el bienestar de quien sufre. El presente artículo tiene como objetivo reflexionar acerca de la actuación del personal de salud y la compasión como elemento clave de la práctica profesional en las instituciones sanitarias. Aunque la compasión es aparentemente intuitiva, persiste una brecha entre lo que los pacientes consideran un pilar de la atención de calidad y la capacidad de los proveedores de la atención médica para brindar este componente esencial. Se concluye que la compasion es una palabra que se encuentra inmersa en el personal de la salud, sin embargo, es un concepto complejo y polisemico, que requiere ser estudiado a la luz de lo que significa y conceptualizan los pacientes, familiares y profesionales de la salud. El modelaje de actitudes compasivas durante la formaciòn, podrian contribuir a cambios positivos en la atenciòn del paciente; en consecuencia al cuidado humanizado.

> Palabras claves: Empatía, personal de salud, atención de enfermería (DeCS, Bireme) 


\section{Compassion as a key element}

of professional practice in

health institutions

\section{, Abstract}

The practice of health professionals is based on an interpersonal relationship, it requires sensitivity and empathy, it is not a mere mechanical activity or an act on the patient or family, it is an activity where the parties are involved in the search for relief of the suffering and well-being of the sufferer. This article aims to reflect on the performance of health personnel and compassion as a key element of professional practice in health institutions. While compassion is seemingly intuitive, there remains a gap between what patients consider to be a pillar of quality care and the ability of healthcare providers to deliver this essential component. It is concluded that compassion is a word that is embedded in health personnel, however, it is a complex and polysemic concept, which needs to be studied in the light of what it means and conceptualize patients, relatives and health professionals. The modeling of compassionate attitudes during training could contribute to positive changes in patient care; as a result of humanized care.

, Keywords: Empathy, health personnel, nursing care (DeCS, Bireme) 


\section{, Introducción}

En la cotidianidad de toda persona, existen situaciones que en un momento determinado influyen para que exista un desequilibrio en su organismo, bien sea por procesos agudos o crónicos, siendo necesario acudir a las instituciones sanitarias en busqueda de la recuperación de la salud. El escenario que enfrenta día a día la persona hospitalizada en la institución sanitaria exige a los profesionales de la salud: médicos y enfermeras, proporcionar atención integral. Esta idea lleva a considerar, que el equipo que labora en la institución sanitaria debe poseer un perfil enmarcado en una filosofía integradora con: valores, destrezas, conocimientos científicos y tecnológicos.

Lo expuesto anteriormente conlleva a reconocer a las instituciones sanitarias como escenarios propicios para ayudar al prójimo, y las acciones de los profesionales de la salud particularmente médicos y enfermeras, podrían interpretarse como actos de asistencia ante el sufrimiento del otro. Los académicos, los profesionales de la salud y los pacientes de todas las disciplinas sanitarias han coincidido en que la compasión en los servicios de salud es un componente necesario y fundamental de la curación(1).

Aunque la compasión parece intuitiva e innata en el personal de salud, se observa en el contacto con el paciente y la familia, actos que se alejan de la compasión. La falta de atención compasiva puede llevar al sufrimiento del paciente y a malos resultados(2), motivo por el cual la compasión en la atención médica ha recibido una atención significativa; a escala internacional, ha surgido preocupación por su ausencia durante las interacciones clínicas(3). Los pacientes y familiares perciben una falta de compasión en su experiencia de atención médica; en consecuencia, pueden tener más eventos adversos, un peor manejo de los síntomas y es más probable que presenten quejas y demanden por negligencia(4). Los pacientes sienten que si los profesionales de la salud, particularmente las enfermeras pudieran ser testigos de su propio comportamiento sin compasión, esto podría alentarlas a cambiar(5).

No cabe dudas, la práctica clínica es una realidad altamente exigente, mucho se espera de sus profesionales y también mucho han de sufrir usuarios y pacientes para recuperar la salud perdida o quebrantada(6). La compasión debe tratarse como un elemento fundamental de una atención médica eficaz, siendo citada no solo como un componente necesario para aumentar la confianza del paciente en sus profesionales de la salud, sino también como un componente crítico de la curación(7). Esto implica reflexionar sobre la actuación del personal de salud (médicos y enfermeras) y la compasión como elemento clave de la práctica profesional en las instituciones sanitarias.

\section{Pero, ¿Qué es la compasión?}

El término de compasión es polisémico, ha sido acuñado por varios autores y desde diversos enfoques. Etimológicamente, las raíces latinas de la palabra 'compasión' incluyen el verbo 'patior' (sufrir) y el afijo 'cum' (con)(2). El concepto de compasión tiene sus orígenes en la palabra compassǐo, -ōnis que a su vez proviene del griego ı४зпа́னiṬa (sympatheīa). Asimismo, en latín pathīa cuyo significado esta relacionado con el sufrimiento; es decir, en resumen compasión significa sufrir con (8). Por su parte, la Real Academia Española, define la compasion como el sentimiento de conmiseración y lástima que se tiene hacia quienes sufren penalidades o desgracias(9).

La definición más primitiva de compasión se atribuye a Aristóteles (eléos), quien la presenta como un sentimiento de tristeza causado por la vista de 
algún mal destructivo o penoso sobre quien no lo merece y podríamos esperar que cayese sobre nosotros o alguien que nos pareciese próximo(10). Asimismo, puede ser apreciada como un acto de bondad básica, con una profunda conciencia del sufrimiento de uno mismo y otros seres vivos, junto con el deseo y el esfuerzo para aliviarlo (11). Puede considerarse como un acto de bondad cuyo empeño reside no en compartir una condición común, sino en alejar la desgracia y la miseria para que no tengan lugar, alejando la amenaza de la muerte (12).

Al respecto, el Dalai Lama manifiesta que las grandes religiones del mundo hacen hincapié en la importancia que tiene el cultivo de la compasión, destaca que no es un asunto religioso, es un asunto humano; no es un lujo, es algo esencial para nuestra propia paz, estabilidad mental y supervivencia humana (13). En este sentido, si la compasión involucra la capacidad para captar y compartir el sufrimiento de los demás, necesariamente los profesionales de la salud tienen el deber moral y ético ante el Estado, la institución, la sociedad y ellos mismos; como parte de la ética profesional, de acercarse al mundo del otro para comprenderlo y desde allí fortalecerlo en la experiencia significativa de la hospitalización.

La compasión constituye un valor, permite que todos sean tratados con igualdad. Es un efecto que surge de la simpatía, la tristeza, la comprensión y la empatía frente a aquellos que se encuentran en una situación peor que la propia (14). La compasión comprende dos dimensiones centrales: la primera es el compromiso definido como la sensibilidad y la conciencia de la presencia de sufrimiento y sus causas. La segunda dimensión es conocida como el alivio o la mitigación y consiste en la motivación para llevar a cabo acciones concretas con el fin de aliviar el sufrimiento (15).
En este sentido, la compasión puede entenderse como un proceso cognitivo, afectivo y conductual, consta de los siguientes cinco elementos referidos tanto al yo como a los demás: 1) Reconocer el sufrimiento; 2) Comprender la universalidad del sufrimiento en la experiencia humana; 3) Sentir empatía por quien sufre y conectarse con la angustia (resonancia emocional); 4) Tolerar los sentimientos incómodos que surgen en respuesta a la persona que sufre (por ejemplo, angustia, ira, miedo) permaneciendo abiertos y aceptando a la persona que sufre; y 5) Motivación para actuar / actuar para aliviar el sufrimiento(16). En concordancia con lo planteado, se establece la estrecha relación entre la compasión y el cuidado como elementos inseparables para la preservación de la especie humana.

También, se ha demostrado científicamente los beneficios de la compasión para la salud: regula las emociones, mejora las relaciones interpersonales y sociales, influye en la fisiología y la activación cerebral, entre otros aspectos. A la luz de estos importantes beneficios, ahora se han desarrollado psicoterapias e intervenciones que tienen como objetivo específico cultivar la compasión (17). En este sentido, se puede apreciar que la compasión trasciende el componente ético - moral a los espacios del conocimiento científico y los beneficios a la salud de la persona.

\section{¿Cómo se relaciona la compasión con los profesio- nales de enfermería y los médicos?}

El cuidado es una vivencia ancestral esencial, vinculada intrínsecamente a la adaptación y las diferentes fases evolutivas del ser humano, donde el cuidado puede ser demostrado y practicado solo interpersonalmente e involucra a la persona cuidadora en este caso el equipo de salud y la persona receptora del cuidado, el paciente. Tanto en el trabajo con pacientes como en nuestra vida 
diaria, el primer gesto compasivo consiste en ver realmente al otro y reconocerlo en su humanidad. A menudo esa cualidad de atención en sí misma, ese reconocimiento de humano a humano, es capaz de aliviar una buena parte del sufrimiento (18).

Por su propia naturaleza, el cuidado determina dos significados relacionados intrínsecamente: actitud de desvelo, de solicitud y de atención para con el otro. El cuidado es ayudar a otra persona a cuidarse por sí misma, y se convierta en responsable de su propia vida (19). Los humanos son seres cuidadores; por tanto, cuidar es un distintivo universal y vital para la sobrevivencia humana. Tal habilidad es influida por nuestra propia experiencia de cuidarnos a nosotros mismos y de ser cuidados por otros (20).

Heidegger, M., el filósofo por excelencia del cuidado, reconoce que éste es una constitución ontológica, siempre subyacente a todo lo que el ser humano emprende, proyecta y hace (21). Por su parte, Boff, L., estima que el cuidado significa desvelo, solicitud, diligencia, celo, atención, buen trato (22). El cuidado es la máxima expresión de comunicación entre los seres vivos, pues trasciende más allá de la simple relación entre humanos, por cuanto exige la presencia significativa de la persona que cuida y de la persona receptora del mismo.

Diferentes teorizantes hacen énfasis en el cuidado como esencia de la Enfermería. Watson, J. prioriza al humano con necesidad de cuidado y amor, éstos a menudo han de ser vistos como lo máximo, pues la humanidad está para sobrevivir, lo que implica que los seres humanos necesitamos llegar a ser más cuidadosos, más afectivos, más amorosos y compasivos. El cuidado humano efectivo puede ser demostrado y practicado interpersonalmente dentro de un contexto transpersonal; trasciende a cada ser humano, y se mueve en círculos concén- tricos del yo hacia el otro, al medio ambiente, a la naturaleza y al universo (23).

Por su parte, Leininger, M. concibe el cuidado como actividades expertas, procedimientos y decisiones relacionados con el apoyo a la gente; refleja los atributos de conducta como: de apoyo, compasivos, protectores, de auxilio, educativos, pendientes de las necesidades, de los valores y metas del individuo o del grupo a quienes se asiste (20). El cuidado profesional encarna las metas cognoscitivas e intencionadas, los procedimientos y los actos de los profesionales o de los grupos que ayudan a otros; expresan actitudes, acciones de interés hacia los demás, para apoyar a su bienestar, aliviar sus trastornos y encontrar las necesidades obvias o previstas.

El cuidado compasivo se puede demostrar con palabras, pero también con una actitud silenciosa, solidaria y respetuosa(24). Los médicos deben ser compasivos y empáticos en el cuidado de los pacientes (25). La correlación entre la empatía de los profesionales de la salud y la recuperacin, la satisfacción y los resultados del tratamiento del paciente están bien establecida. Además, la satisfacción profesional de los proveedores de atención médica también se correlaciona con la empatía (26). Para los pacientes y los familiares, contar con un equipo de salud presente, cercano, empático y compasivo supone un alivio tremendo en la experiencia que están viviendo. Hoy en día sabemos que la actitud de los profesionales tiene el poder de aumentar o disminuir el dolor en los pacientes y la angustia en los familiares (18).

Los profesionales de enfermería mediante el cuidado tienen la oportunidad de estar frente a la persona que requiere acciones cuidadoras, en las cuales se demuestran las habilidades de presencia significativa a través del acercamiento al otro en el marco de una relación interpersonal basada en la 
confianza y el cuidado significativo que trasciende la ejecución de acciones. El cuidado involucra un proceso de aprendizaje de saber escucharnos a nosotros mismos para poder escuchar atentamente al otro. Saber cuándo tocar y la manera cómo hacerlo, transmite seguridad, afecto, sinceridad, calor entre muchos otros sentimientos; el tocar muchas veces dice más que las palabras, y esto es importante para la persona receptora de cuidado. El ver es otra actitud cuidadora, determina la presencia significativa del profesional de enfermería, quien debe aprender a interpretar en cada expresión kinestésica lo que el paciente intenta manifestar y siente en el momento del contacto interpersonal (27). Por consiguiente, el profesional de enfermería debe apoyar a la persona cuidada por medio de actitudes y acciones que muestren interés por su bienestar y su aceptación como persona que piensa, siente, y padece. Evitar considerarlo como un ser cosificado, cuyas respuestas se deben a un desequilibrio biológico manifestado por signos y síntomas.

Por su parte, el médico debe empeñarse en el conocimiento integral de lo que justifica su razón de ser: el hombre. La actual situación mundial, muestra que debemos imponernos como trabajadores de la salud, un reto ético que conduzca a la construcción de un hombre cada vez más humano, más racional, más comunicativo, más afectivo, más respetuoso con la realidad, mas dueño de sí mismo y responsable de sus actos ante los demás. Asimismo, en el ejercicio de su profesión, el médico en innumerables ocasiones va a encontrarse cara a cara con el dolor y con el sufrimiento lo cual requiere estar capacitado para servir a quienes sufren, muchos de los cuales tendrán enfermedades incurables. En esta solidaridad humana se origina probablemente "la razón de ser" de la medicina como arte de aminorar el sufrimiento ajeno lo cual es uno de los desafíos de la medicina actual(28).
En las instituciones asistenciales, se observa un "enfriamiento" en el amor, la solidaridad, la compasión y la empatía, siendo imperativo que las profesiones de la salud: enfermeras y médicos, reflexionen acerca de la atención humanizada, que involucre seres cuidadores, compasivos y afectivos. Tanto el medico como la enfermera (o) como integrantes fundamentales del equipo de salud, deben incluir en su práctica profesional actividades para proteger la dignidad humana, así como conocimientos y sensibilidad hacia lo importante para los pacientes. Ahora bien, ¿cómo se articula la dignidad humana con la compasión?, se articula desde el punto de vista del respeto por el valor inherente de cada ser humano, entendiendo la dignidad humana como el derecho de la persona a ser respetada y valorada como ser individual y social, con sus características y condiciones particulares, por el solo hecho de ser persona(29).

La compasión no es signo de debilidad, al contrario, sólo personalidades dotadas de fuertes y arraigadas convicciones humanistas pueden hacerse cargo de la compasión como virtud, con todas las consecuencias políticas que ello conlleva(30). En este sentido, la compasión reconoce al otro como un ser integral a quien se le respeta la vida y sus derechos. Una relación basada en la dignidad y el respeto da como resultado un cuidado compasivo, visto como el principio fundamental en cómo cuidar a los demás(31). Respuestas compasivas a otros son básicas en la naturaleza humana.

\section{¿Cuál es la importancia de la compasión en la for- mación de los profesionales de la salud?.}

La compasión es la base de una buena atención médica. Reconoce las preocupaciones, angustia y sufrimiento de los pacientes y familiares, con la finalidad de establecer medidas para aliviar el sufrimiento. Se basa en la escucha, respeto, empatía, comunicación, habilidades interpersonales, 
conocimiento, comprensión de la vida y preferencias del paciente. En esencia, significa tratar a los pacientes como personas, no solo como enfermedades(32). A pesar de la prevalencia de la compasión en el discurso actual, existe una disparidad creciente entre los beneficios curativos de la compasión en los servicios de salud y los comentarios tanto de los usuarios como proveedores de servicios sobre la falta de compasión en el sistema de salud actual(1).

La compasión parece desarrollarse de manera óptima a través del aprendizaje experiencial y reflexivo, tanto en el contexto de la formación clínica de los estudiantes como en las experiencias personales (33). En el contexto de la formación clínica, la percepción de los alumnos es que la enseñanza de la medicina en el momento actual carece de una dimensión humana (34). Se multiplican las quejas de que el modelo médico científico no preparaba a los profesionales para comprender adecuadamente, atender integralmente y cuidar humanamente a los pacientes (35). Surgen planteamientos enfocados hacia la selección de los candidatos, aunque muchas veces se reciben alumnos entusiasmados, con clara vocación, y de algún modo se desaniman por las exigencias incongruentes durante la formación (36). En consecuencia, el nivel de empatía puede verse influido por las intervenciones educativas inculcadas a los estudiantes durante todo el plan de estudios, incluida la formación tanto didáctica como experiencial(25).

Hoy en día sabemos que la actitud de los profesionales tiene el poder de aumentar o disminuir el dolor en los pacientes y la angustia en los familiares. En este sentido, el desarrollo de 'culturas compasivas' en los equipos de salud es una situación donde todos ganan: disminuye el estrés y burnout de los profesionales, mientras los pacien- tes y familiares reciben el trato humano que merecen y necesitan (18). Se requieren políticas para fomentar la compasión en los estudiantes de enfermería y medicina, pero no existen pautas para las instituciones educativas. Puede resultar útil centrarse en el dominio del aprendizaje afectivo, facilitar la reflexión e incorporar indicadores de atención compasiva en las experiencias de aprendizaje clínico, es importante integrar e investigar acerca de la compasión en los planes de estudios para fomentarla en los estudiantes (37). La educación relacionada con la expresión de la compasión debe articular tanto la subjetividad como la ambigüedad del término y examinar la relación entre la compasión y el sufrimiento (38).

Para muchos médicos en formación y residentes, la compasión del médico, su actitud comunitaria, constituyen un deber de orden ético. El médico en formación, aun el residente, se inician en el conocimiento de una profesión para la cual la dedicación al servicio del enfermo es el motivo de su existencia (39). La educación médica debe abarcar tanto formación técnica como moral, las cuales son verdaderamente inseparables(40). En cuanto a las materias de estudio, las humanidades médicas tienen que abarcar todo lo que nos hace humanos y todo lo que mejora nuestra humanidad(35), esta es una cualidad que debe asociarse a integridad, devoción y capacidad y, a menos que no sean cabalmente desarrolladas en los años formativos, es poco probable que emerjan espontáneamente cuando las demandas del ejercicio profesional y las frustraciones de la vida sacudan la humanidad de estos profesionales (41).

\section{) Consideraciones Finales}

Aunque la compasión parece intuitiva e innata, los profesionales de la salud deben desarrollarla y manifestarla a través de la acción. No basta con 
pensar que médicos y enfermeras son compasivos por naturaleza, es necesario fomentar proyectos de formación que ayuden a desarrollar prácticas compasivas en el ejercicio profesional, considerando los beneficios psicológicos y fisicos que genera a quien práctica y recibe actos compasivos. En este sentido, tanto médicos como enfermeras deben reunirse para reflexionar acerca de la práctica que realizan, de manera que puedan entenderse y generar acciones que contribuyan a mostrar interés y respeto por el usuario. La compasión es un valor ético-moral, pero también una habilidad que debe desarrollar quien se dedica al cuidado y cura del desvalido..

Los docentes y personal de salud son modelos para los estudiantes en formación, quienes a través del ejemplo pueden demostrar actos compasivos, esto a su vez sería altamente gratificante al sentirse util ayudando a la otra persona que esta en situacion vulnerable. Esta comprobado, cuando una persona es compasiva influye positivamente en el aspecto fisico, emocional y mental, lo cual quedara reflejado en la practica profesional futura.

La compasion es una palabra que se encuentra inmersa en el personal de la salud, sin embargo, es un concepto complejo y polisemico, que requiere ser estudiado a la luz de lo que significa y conceptualizan los pacientes, familiares y profesionales de la salud. A pesar de la considerable discusión sobre el tema, esta es todavía un área de estudio incipiente.

\section{> Referencias Bibliográficas}

1. Wiljer, David, et al. Defining compassion in the digital health age: protocol for a scoping review. BMJ open, 2019, vol. 9, no 2, p. e026338. https://doi.org/10.1136/bmjopen-2018-026338. [Acceso 22.02.21]. Disponible en: https://www.ncbi. nlm.nih.gov/pmc/articles/PMC6398782/.
2. Bickford, Ben, et al. Understanding compassion for people with dementia in medical and nursing students. BMC medical education, 2019, vol. 19, no 1, p. 1-8. https://doi.org/10.1186/s12909-0191460-y. [Acceso 12.02.21]. Disponible en: https:// www.ncbi.nlm.nih.gov/pmc/articles/PMC6347776/.

3. Tierney, Stephanie; Bivins, Roberta; Seers, Kate. Compassion in nursing: Solution or stereotype?. Nursing inquiry, 2019, vol. 26, no 1, p. e12271. https://doi.org/10.1111/nin.12271. [Acceso 10.02.21]. Disponible en: https://www.ncbi.nlm.nih.gov/ pmc/articles/PMC6492101/.

4. Sinclair, Shane, et al. Healthcare providers perspectives on compassion training: a grounded theory study. BMC Medical Education, 2020, vol. 20, no 1, p. 1-13. Doi: 10.1186 / s12909-020-02164-8. [Acceso 21.01.21]. Disponible en: https://www.ncbi. nlm.nih.gov/pmc/articles/PMC4717626/

5. Bramley, L. y Matiti, M. (2014). How does it really feel to be in my shoes? Patients' experiences of compassion within nursing care and their perceptions of developing compassionate nurses. Revista de enfermería clínica, 23 (19-20), 27902799. https://doi.org/10.1111/jocn.12537. [Acceso 25.01.21]. Disponible en: https://www.ncbi.nlm.nih. gov/pmc/articles/PMC4263156/

6. Jiménez, A., Triana, M., y Washburn, J. (2002). Compasión y salud. Isegoría, (27), 211-223. https:// doi.org/10.3989/isegoria.2002.i27.563. [Acceso 12.02.21]. Disponible en: http://isegoria.revistas. csic.es/index.php/isegoria/article/view/563.

7. Strudwick, Gillian y col. Delivery of compassionate mental health care in a digital technologydriven age: protocol for a scoping review. Abierto de BMJ , 2019, vol. 9, no 7, pág. e027989. https:// doi.org/10.1136/bmjopen-2018-027989. [Acceso 16.02.21]. Disponible en: https://www.ncbi.nlm.nih. gov/pmc/articles/PMC6661656. 
8. Hernández E. Danilo K. Visión históricovalorativa de la compasión: de la Antigüedad a la Modernidad. 2014. [Acceso 15.03.21]. Disponible en http://cybertesis.unmsm.edu.pe/bitstream/cybertesis/3858/1/Hernandez_ed.pdf

9. Real Academia Española (2020). Diccionario de la lengua española. [Acceso 11.01.21]. Disponible en: https://dle.rae.es/compasi\%C3\%B3n

10. Aristóteles. (2004). Retórica. Madrid: Alianza Editorial.

11. Gilbert, Paul. Introducing compassion-focused therapy. Advances in psychiatric treatment, 2009, vol. 15, no 3, p. 199-208. doi: Disponible en: https://bit.ly/30lu1Do

12. Sinclair, Shane, et al. Compassion: a scoping review of the healthcare literature. BMC palliative care, 2016, vol. 15, no 1, p. 1-16. https://doi. org/10.1186/s12904-016-0080-0. [Acceso 11.02.21]. Disponible en: https://www.ncbi.nlm.nih.gov/ pmc/articles/PMC4717626

13. Lama D. El arte de vivir en el nuevo milenio [Internet]. Dharamsala: Editorial: Grijalbo Mondadori, editor. 1999. 43 p. Disponible en: https://jesuitas.lat/uploads/el-arte-de-vivir-en-el-nuevomilenio-pag-30-77/DALAI LAMA - 2010 - EL ARTE DE VIVIR EN EL NUEVO MILENIO PAG. 30-77.pdf.

14. Significado de la compasion [Internet]. Disponible en: www. Significados.com.

15. Alfonso Martha. Compasión en la práctica clínica: una revisión conceptual y empírica. [Acceso 11.01.21]. Disponible en: http:// www.fundacionomie.org/wp-content/uploads/ Art\%C3\%ADculo-Compasi\%C3\%B3n-en-laPr\%C3\%A1ctica-Cl\%C3\%ADnica-Marta-Alonso-Revista-Actas-de-Psiquiatr\%C3\%ADa.pdf..

16. Strauss, Clara, et al. What is compassion and how can we measure it? A review of definitions and measures. Clin Psychol Rev. 2016 Jul; 47: 15-27. doi: 10.1016/j.cpr.2016.05.004. [Acceso 20.02.21]. Disponible en: https://www.sciencedirect.com/science/article/pii/S0272735816300216?via\%3Dihub.

17. Kirby, James N. Intervenciones de compasión: los programas, la evidencia y las implicaciones para la investigación y la práctica. Psicología y psicoterapia: teoría, investigación y práctica. 2017, vol. 90, no 3, pág. 432-455. https://doi.org/10.1111/ papt.12104. [Acceso 10.06.21]. Disponible en: https://bpspsychub.onlinelibrary.wiley.com/doi/ abs/10.1111/papt.12104

18. Brito Pons Gonzalo. Entrevista: La compasión en profesionales de la salud [Internet]. Cultivar la mente. Salud mental y pràcticas contemplativas. [Acceso 21.02.21]. Disponible en: https:// cultivarlamente.com/entrevista-la-compasionen-profesionales-de-la-salud/

19. Mayeroff, M. (1971). A Arte de Servir Ao Próximo Para Servir a si Mesmo. Brasil: Editorial Record. Traductora: Cristina Carvalho Boselli. Pág. 33-44..

20. Leininger, M. (1991). Culture Care Diversity and Universality: A Theory of Nursing. New York: National League for Nursing Press. Pág. 36.

21. Heidegger, M. (1991). El Ser y el Tiempo. México: Fondo de Cultura Económica. Traductor: José Gao. Pág. 220

22. Boff, L. (1999). Saber Cuidar. Ética do Humano. Compaxaio pela Terra. Petrópolis, Brasil: Editora Vozes. Pág. 91.

23. Watson, J. (1996). Teoría del Cuidado Transpersonal. Nueva York. Traducido por: Jorge Gómez. Pág.15 - 17.

24. Perez-Bret E, Altisent R, Rocafort J. Definition of compassion in healthcare: a systematic literatu- 
re review. Int J Palliat Nurs. 2016 Dec;22(12): 599-606. doi: 10.12968/ijpn.2016.22.12.599. [Acceso 11.02.21]. Disponible en: https://www.magonlinelibrary. com/doi/abs/10.12968/ijpn.2016.22.12.599?rfr_ dat $=c r \_p u b++0$ pubmed\&url_ver $=Z 39.88-2003 \&$ rfr_ id=ori\%3Arid\%3Acrossref.org

25. Ratka A. Empathy and the Development of Affective Skills. Am J Pharm Educ. 2018 Dec; 82 (10):7192. doi: 10.5688/ajpe7192. [Acceso 22.01.21]. Disponible en: https://www.ncbi.nlm.nih.gov/ pmc/articles/PMC6325458/

26. Stepien KA, Baernstein A. Educating for empathy. A review. J Gen Intern Med. 2006 May; 21 (5): 524-30. doi: 10.1111/j.1525-1497.2006.00443.x. [Acceso 19.01.21]. Disponible en: https://www.ncbi.nlm. nih.gov/pmc/articles/PMC1484804/.

27. Guevara B., Evies A., Rengifo J., Salas B., Manrique D., Palacio C.. Nursing care: an integrating vision in times of crisis. Enferm. glob. [Internet]. 2014 Ene 13( 33 ): 318-327. [Acceso 21.03.21] Disponible en: http://scielo.isciii.es/scielo.php?script=sci_ arttext\&pid=S1695-61412014000100016\&lng=es.

28. Yegüez, F. (2003). Dolor y Sufrimiento Humano. Valencia, Venezuela: Material mimeografiado. Facultad de Ciencias de la Salud. Universidad de Carabobo.

29. Casas L. Luisa F. Diseño de una estrategia didáctica de enseñanza integral de la alteridad, la empatía y el valor de la compasión para profesionales de la salud. 2020. [Acceso 25.02.21]. Disponible en: https://ciencia.lasalle.edu.co/doct_educacion_sociedad/41/

30. Bueno A. Miryam Inmaculada, et al. Compasión, valor ético del profesional enfermero: Estudio etnográfico en el área de salud de Soria. 2018. Tesis Doctoral. Universitat de Barcelona. [Acceso 03.02.21]. Disponible en: https://www.tdx.cat/ handle/10803/593504.

31. Krausé, S. S., Minnie, C. S., y Coetzee, S. K. (2020). The characteristics of compassionate care during childbirth according to midwives: a qualitative descriptive inquiry. BMC pregnancy and childbirth, 20 (1), 304. https://doi.org/10.1186/ s12884-020-03001-y. [Acceso 21.01.21]. Disponible en: https://www.ncbi.nlm.nih.gov/pmc/articles/ PMC7236148/

32. Hofmeyer A, Taylor R, Kennedy K. Fostering compassion and reducing burnout: How can health system leaders respond in the Covid-19 pandemic and beyond? Nurse Educ Today. 2020 Nov;94:104502. doi: 10.1016/j.nedt.2020.104502. [Acceso 25.01.21]. Disponible en: https://www.ncbi. nlm.nih.gov/pmc/articles/PMC7295512

33. Sinclair, S., Norris, J. M., McConnell, S. J., Chochinov, H. M., Hack, T. F., Hagen, N. A., McClement, S., \& Bouchal, S. R. (2016). Compassion: a scoping review of the healthcare literature. BMC palliative care, 15, 6. https://doi.org/10.1186/s12904-0160080-0. [Acceso 20.01.21]. Disponible en: https:// www.ncbi.nlm.nih.gov/pmc/articles/PMC4717626/

34. Millán Núñez-Cortés, Jesús. Humanización de la medicina, medicina humanizada, medicina humanista: ¿ de qué estamos hablando?. Educ. med.(Ed. impr.), 2018, p. 131-132. [Acceso 23.01.21]. Disponible en:https://pesquisa.bvsalud.org/por$\mathrm{tal} / \mathrm{resource} / \mathrm{pt} / \mathrm{ibc}-194873$.

35. González, Miguel Ángel Sánchez. El humanismo y la enseñanza de las humanidades médicas. Educación médica, 2017, vol. 18, no 3, p. 212-218. [Acceso 11.02.21]. Disponible en: https:// www.sciencedirect.com/science/article/pii/ S1575181317300463

36. Moreto, Graziela; Blasco, Pablo González; Piñero, Agustina. Reflexiones sobre la deshumaniza- 
ción de la educación médica: empatía, emociones y posibles recursos pedagógicos para la educación afectiva del estudiante de medicina. Educación Médica, 2018, vol. 19, no 3, p. 172-177. [Acceso 14.01.21]. Disponible en: https://www.sciencedirect.com/science/article/pii/S1575181317300141

37. Younas A, Maddigan J. Proposing a policy framework for nursing education for fostering compassion in nursing students: A critical review. J Adv Nurs. 2019 Aug;75(8):1621-1636. doi: 10.1111/ jan.13946. [Acceso 22.01.21]. Disponible en: https:// onlinelibrary.wiley.com/doi/abs/10.1111/jan.13946.

38. Durkin J, Usher K, Jackson D. Embodying compassion: A systematic review of the views of nurses and patients. J Clin Nurs. 2019 May;28(910):1380-1392. doi: 10.1111/jocn.14722. [Acceso 12.01.21]. Disponible en: https://onlinelibrary.wiley.com/doi/abs/10.1111/jocn.14722.

39. Tamayo M., Salvador. Schooling for the physicians we need. Rev cubana med [online]. 2015, vol.54, n.1, pp.1-5. [Acceso 2021-03-29]. Disponible en: $\quad$ <ttp://scielo.sld.cu/scielo.php?script=sci_ arttext\&pid=S0034-75232015000100001\&lng=es\&nr $\mathrm{m}=$ iso>. ISSN 0034-7523.

40. Marqués, Lumaira Maria Nascimento Silva da Rocha, et al. ¿Cuáles son los valores esenciales para la formación médica?. Revista Bioética, 2020, vol. 28, no 4, p. 693-703. [Acceso 14.01.21]. Disponible en: https://www.scielo.br/scielo.php?pid=S198380422020000400693\&script=sci_arttext\&tlng=es

41. Mejía, María A.; et al. Comportamiento empático en los estudiantes de las ciencias de la salud. Universidad de Los Andes Fermentum. Revista Venezolana de Sociología y Antropología, vol. 23, núm. 67, mayo - agosto, 2013, pp. 203-220 Universidad de los Andes Mérida, Venezuela. [Acceso 22.01.21]. Disponible en: https://www.redalyc.org/ pdf/705/70538669005.pdf.. 